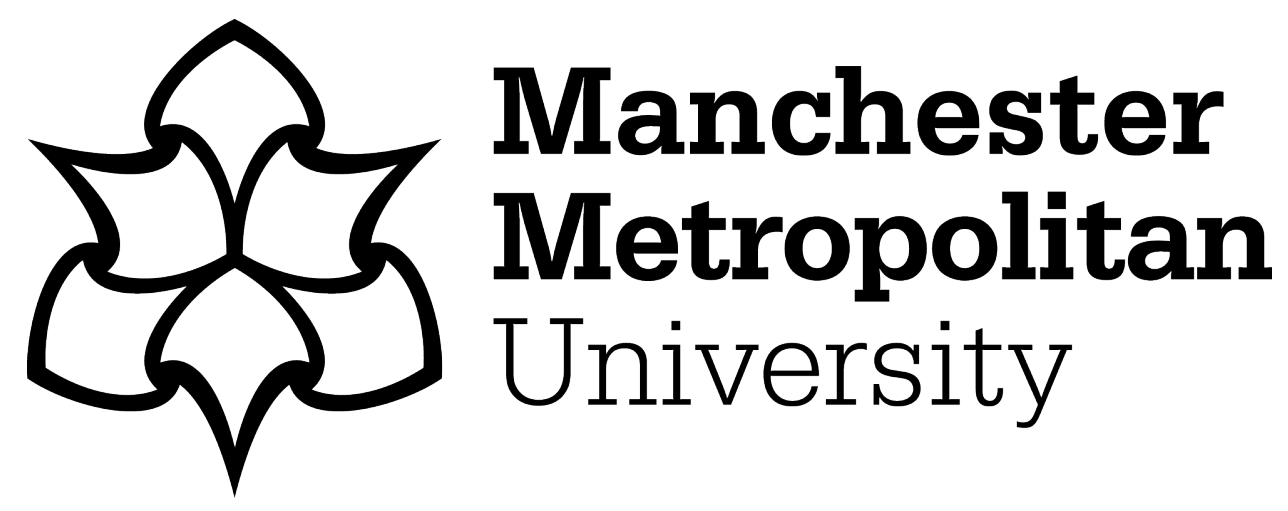

Han, TT, Wang, LN and Potgieter, JH ORCID logoORCID: https://orcid.org/0000-0003-2833-7986 (2019) ZIF-11 derived nanoporous carbons with ultrahigh uptakes for capture and reversible storage of volatile iodine. Journal of Solid State Chemistry, 282. ISSN 0022-4596

Downloaded from: https://e-space.mmu.ac.uk/625141/

Version: Accepted Version

Publisher: Elsevier

DOI: https://doi.org/10.1016/j.jssc.2019.121108

Please cite the published version 


\title{
ZIF-11 derived nanoporous carbons with ultrahigh uptakes for capture and reversible storage of volatile iodine
}

Ting-Ting Han ${ }^{\mathrm{a}, \mathrm{b}}$, Li-Ning Wang ${ }^{\mathrm{c}}$ and J.Herman Potgieter ${ }^{\mathrm{b}}$

${ }^{a}$ Department of Chemistry, Changchun Normal University, Changchun 130032, P.R. China

${ }^{\mathrm{b}}$ Department of Natural Science, Manchester Metropolitan University, Manchester M1 5GD, United Kingdom

${ }^{\mathrm{c}}$ Shenyang Chemical Industry School, Shenyang 110122, P.R. China

Corresponding author

E-mail address: hantt1984@hotmail.com (T.-T. Han)

\begin{abstract}
In this work, three nanoporous carbon materials Z11-700, Z11-800 and Z11-900 were synthesized by a one-step calcination of zeolitic imidazolate framework-11 (ZIF-11) at 700,800 and $900{ }^{\circ} \mathrm{C}$, respectively. The samples obtained were characterized by SEM, PXRD, FT-IR, Raman spectra and $\mathrm{N}_{2}$ adsorption. The results showed that the three ZIF-11 derived samples were micro/mesoporous and had high BET surface areas and pore volumes. Of the synthesized samples, Z11-900 displayed an excellent iodine vapour uptake of $3775 \mathrm{mg} / \mathrm{g}$, which was superior compared with Z11-700 and Z11-800, as well as other reported solid state materials. What is more, the regeneration efficiency of Z11-900 for iodine molecules was near to $90 \%$ (3672 mg/g) after three cycles of reuse. The easy operation, highly-effective and recyclable features enable the ZIF-11 derived nanoporous carbons to be a promising adsorbent for applications in environmental remediation.
\end{abstract}


Key words: zeolitic imidazolate framework-11; porous carbon; iodine adsorption; regeneration

\section{Introduction}

With the rapidly growing needs for energy sources and global warming, nuclear power is becoming one of the most feasible and prominent alternative sources as a green, non-carbon emitting and reliable energy source [1,2]. However, radioactive iodine is one of the volatile radionuclide fission products in nuclear waste, which can cause pollution and poses an exceptional long-term risk due to its long radioactive half-lives ( 8 days for ${ }^{131} \mathrm{I}$ and $1.57 \times 10^{7}$ years for ${ }^{129} \mathrm{I}$ ), high volatility, easily dissolution in water and adverse damage to human metabolic processes and the environment [3]. At present, a typical method of capturing iodine is using porous materials (such as activated carbon, silica and zeolites) impregnated with an organic amine (such as triethyl diamine) as adsorbents [4-5]. These adsorbents display a high affinity for radioactive iodine, however, their shortcomings could not be ignored. For example, sublimation of organic amine occurs easily, leading to a reduction in adsorption efficiency [6]. In addition, the adsorbents modified with organic amine is prone to aging, which could cause the desorption of captured iodine and secondary pollution. In the past few years, porous organic polymers (POPs) and metal-organic frameworks (MOFs) have proved to be candidates for capturing iodine due to their easy operation, low energy consumption and excellent adsorption performance [7-10]. However, the limited recyclability and poor cycling stability reduce their employment as ideal adsorbents. Graphene has also been exploited to adsorp iodine because of its high specific surface area and controllable functional groups [11]. The research results yielded graphene powder with an adsorption capacity of $850 \mathrm{mg} / \mathrm{g}$. Graphene based nanomaterials co-doped with $\mathrm{S}, \mathrm{N}$ like graphene aerogel displayed a maximum iodine 
uptake capacity of $999 \mathrm{mg} / \mathrm{g}$ [12]. Graphene is not quite an ideal iodine adsorbent in terms of the adsorption capacity. Therefore, there is still an urgent and vital necessity to search for alternative effective materials to capture volatile radionuclide iodine $[13]$.

In recent years, porous carbon adsorbents have drawn considerable attention in the fields of capture and storage of gaseous compounds, because of their large surface areas, highly developed porosities, physicochemical robustness and recyclability [14]. Generally, there are three synthesis methods to obtain porous carbon materials. The first synthesis strategy is the one-step carbonization of templates such as POPs or MOFs under a flow of inert gas [15-16]. The resulting samples could retain similar morphology, size and pore properties as the precursors. Therefore, choosing or designing an appropriate template to carbonize so as to capture target molecules, is extremely important. Sometimes pyrolysis together with a subsequently acid washing procedure was used to optimize the porous carbon samples [17]. Furthermore, there are also reports that several porous carbon materials after carbonizing could be further activated by fused potassium hydroxide at higher than $600{ }^{\circ} \mathrm{C}$ under nitrogen gas $[18,19]$. However, using neither acid nor alkali is an optimum option, considering the potential environmental impact. The second preparation technique is carbonizing MOFs and additional carbon sources such as furfuryl alcohol (FA) [20]. This process generally includes two procedures: (i) introduction of FA into the pores of MOFs by incipient wetness or ultrasonic technique; (ii) pyrolyzing the compound FA@MOFs. The obtained carbon materials displayed an outstanding electrochemical performance because of the improvement of power and energy density. The third synthesis method is surface modification for nanostructured carbon such as carbon nanotubes (CNTs) and graphene by hydrothermal treatment, and then combined with a carbonizing 
technique [21-25]. For example, CNTs or graphene could be modified by vanadium by the hydrothermal method, and the resultant materials showed an excellent catalytic performance for oxidative dehydrogenation of propane. [26].

Zeolitic imidazolate frameworks (ZIFs), a subfamily of MOFs, are crystalline three-dimensional (3D) frameworks composed of metal ions and a tetrahedral imidazolate linker [27]. In recent years, ZIFs derived porous carbon materials have become promising candidates in gas storage/separation and liquid adsorption applications due to their uniform framework structures, and high thermal and chemical stabilities [28-32]. ZIF-11 with a RHO topology is constructed from large cages (diameter of $14.6 \AA$ ) connected through small apertures (diameter of $3.0 \AA$ ) to produce a zeolitic structure built by zinc ions and the nitrogen atoms from benzimidazole (bim) anions [33]. ZIF-11 derived porous carbons materials could maintain the apertures of the sacrificial template ZIF-11 and is close to the $3.30 \AA$ kinetic diameter of iodine, which could efficiently contain guest iodine molecules. To date, only limited reports appeared in literature which indicated that ZIFs could capture iodine molecules into their pores [34]. Even fewer studies have reported the capture and storage of iodine molecules via ZIFs derived porous carbons. In view of these facts, three ZIF-11 derived nanoporous carbons Z11-700, Z11-800 and Z11-900 were synthesized by a one-step calcination of ZIF-11 at 700, 800 and $900{ }^{\circ} \mathrm{C}$ under a nitrogen atmosphere, respectively and used to investigate the adsorption of iodine. The structure and composition of the synthesized samples were characterized and analysed by SEM, PXRD, FT-IR, Raman spectra and $\mathrm{N}_{2}$ adsorption. The adsorption performance of Z11-700, Z11-800 and Z11-900 towards iodine molecules, and the recyclability and efficiency of Z11-900 for radioactive iodine adsorption, had been investigated. The easy operation, highly-effective and recyclable features enable the 
ZIF-11 derived nanoporous carbon Z11-900 to be a promising material in applications for environmental remediation.

\section{Experimental}

\subsection{Materials and Methods}

All chemicals and reagents were commercially available and used directly without further purification. Benzimidazole $\left(\mathrm{C}_{7} \mathrm{H}_{6} \mathrm{~N}_{2}, 98.5 \%\right)$, zinc acetate dihydrate $\left(\mathrm{C}_{4} \mathrm{H}_{6} \mathrm{O}_{4} \mathrm{Zn} \cdot 2 \mathrm{H}_{2} \mathrm{O}, 98 \%\right)$, ethanol $\left(\mathrm{C}_{2} \mathrm{H}_{5} \mathrm{OH}, 99.7 \%\right)$, ammonium hydroxide solution $\left(\mathrm{NH}_{3} \cdot \mathrm{H}_{2} \mathrm{O}, 25-28 \%\right)$, methanol $\left(\mathrm{CH}_{3} \mathrm{OH}, 99.5 \%\right)$ and iodine $\left(\mathrm{I}_{2}, 99.8 \%\right)$ were all analytical grade (AR) and purchased from the Macklin company. Toluene $\left(\mathrm{C}_{6} \mathrm{H}_{5} \mathrm{CH}_{3}\right.$, AR) was purchased from the Merck company. Here, we used stable ${ }^{127}$ I instead of the radioactive ${ }^{129} \mathrm{I}$ and ${ }^{131} \mathrm{I}$ ones, because of the nearly identical chemical behaviour between these isotopic iodine elements [14]. A series of physical characterization methods were given in supporting information (SI).

\subsection{Synthesis of ZIF-11}

ZIF-11 was synthesized according to literature reports [35]. Typically, $1.20 \mathrm{~g}$ of benzimidazole (bim) was dissolved in $68.0 \mathrm{~g}$ of ethanol, then $46.0 \mathrm{~g}$ of toluene and $0.6 \mathrm{~g}$ of ammonium hydroxide solution were added to the bim solution. Next, $1.10 \mathrm{~g}$ of zinc acetate dihydrate was added to the above solution. After these additions, the mixture was stirred for $4 \mathrm{~h}$ at room temperature. The resulting white precipitates (ZIF11) were collected by centrifugation at $6000 \mathrm{rpm}$ for $10 \mathrm{~min}$, washed four times with ethanol, and then dried at $80{ }^{\circ} \mathrm{C}$ for $24 \mathrm{~h}$. The structure of the as-synthesized ZIF-11 was characterized by scanning electron microscope (SEM), power X-ray diffraction (PXRD), Fourier transform infrared spectroscopy (FT-IR) and the results were identical with those reported previously [36,37]. (See Figs. S1 S3 in SI)

\subsection{Preparation of nanoporous carbons}


During the carbonizing process, the dried ZIF-11 powders was directly heated to $900{ }^{\circ} \mathrm{C}$ with a heating rate of $5{ }^{\circ} \mathrm{C} / \mathrm{min}$ and maintained at this temperature for $3 \mathrm{~h}$ under a nitrogen flow (ca. $50 \mathrm{~mL} / \mathrm{min}$ ). After cooling down to room temperature, the final black powders was collected and denoted as Z11-900. Z11-700 and Z11-800 were obtained via a similar synthetic method, except that ZIF-11 was pyrolized at 700 and $800^{\circ} \mathrm{C}$, respectively.

\subsection{Iodine uptake experiments}

$40 \mathrm{mg}$ of samples (ZIF-11, Z11-700, Z11-800 and Z11-900) were placed in a pre-weighted glass vessel with an excess of solid iodine and heated together in a sealed container at $75{ }^{\circ} \mathrm{C}$ and ambient pressure. The experimental conditions were similar to the typical nuclear fuel processing conditions. At various time intervals, samples were removed and allowed to cool to room temperature, and then gravimetric measurements were conducted to determine the iodine capture [38]. The iodine adsorption capacity was calculated by the equation: $\left(\mathrm{m}_{1}-\mathrm{m}_{0}\right) / \mathrm{m}_{0}$, where $\mathrm{m}_{0}$ and $\mathrm{m}_{1}$ were the mass of the samples before and after iodine capture.

\subsection{Iodine desorption experiments}

Regeneration of Z11-900 saturated with iodine molecules was conducted by using a solvent desorption technique [39]. The iodine-loaded Z11-900 was dispersed in methanol at room temperature. The colourless methanol gradually changed from light purple to dark brown. Absorbance of the methanol solution was measured to monitor the presence of iodine by UV/Vis spectroscopy. The desorption experiments was accomplished until the amount of the trapped iodine in methanol was unchanged. The regenerated Z11-900 was collected by centrifugation, washed four times with fresh methanol and dried overnight for the next use. Recyclability experiments were performed according to iodine uptake experiments methods. 


\section{Results and discussion}

\subsection{Structural characterization}

As shown as Fig. 1, SEM images confirmed that the obtained Z11-700, Z11-800 and Z11-900 retained a uniform rhombic dodecahedral shape, which is the same as those of the ZIF-11 precursors, (Fig. S1) and had an average size of $\sim 5 \mu \mathrm{m}$ [40]. In addition, the rough surface could also be observed because of the decomposition of the carbon sources during the carbonization [41].

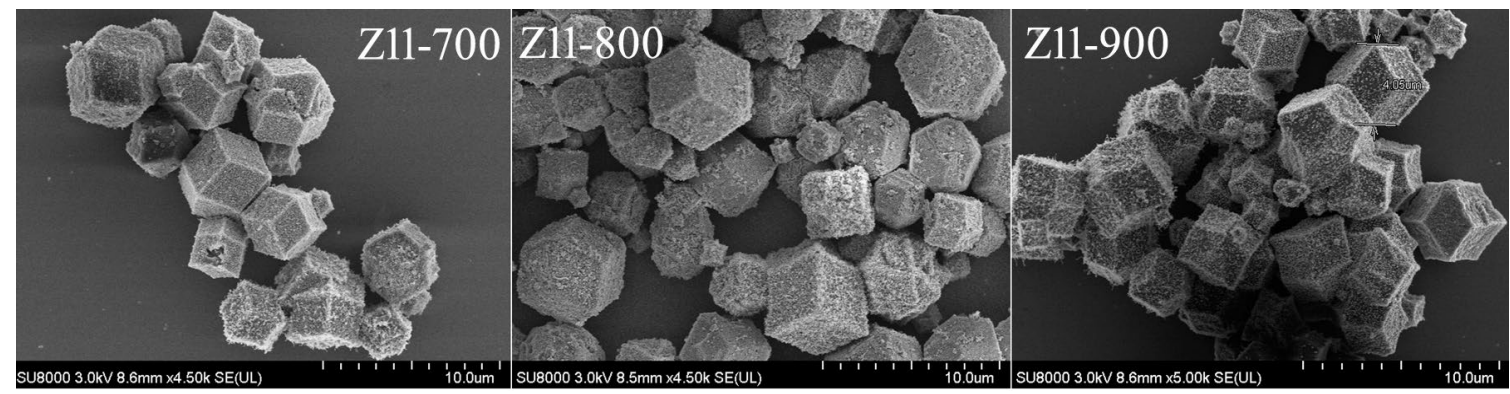

Fig. 1 SEM images of Z11-700, Z11-800 and Z11-900.

As shown as Fig. 2, PXRD patterns of Z11-700, Z11-800 and Z11-900 revealed the presence of graphitic carbon (002) and amorphous carbon (101), corresponding to two broad peaks at about $24^{\circ}$ and $44^{\circ}$, respectively [42]. Any characteristic diffraction of ZIF-11 could not be observed, which confirmed that the ZIF-11 frameworks were carbonized totally. (Fig. S2) The average sizes of samples Z11-700, Z11-800 and Z11-900 were 0.91, 0.90 and $0.90 \mathrm{~nm}$, calculated by Scherrer's equation, respectively [43] 


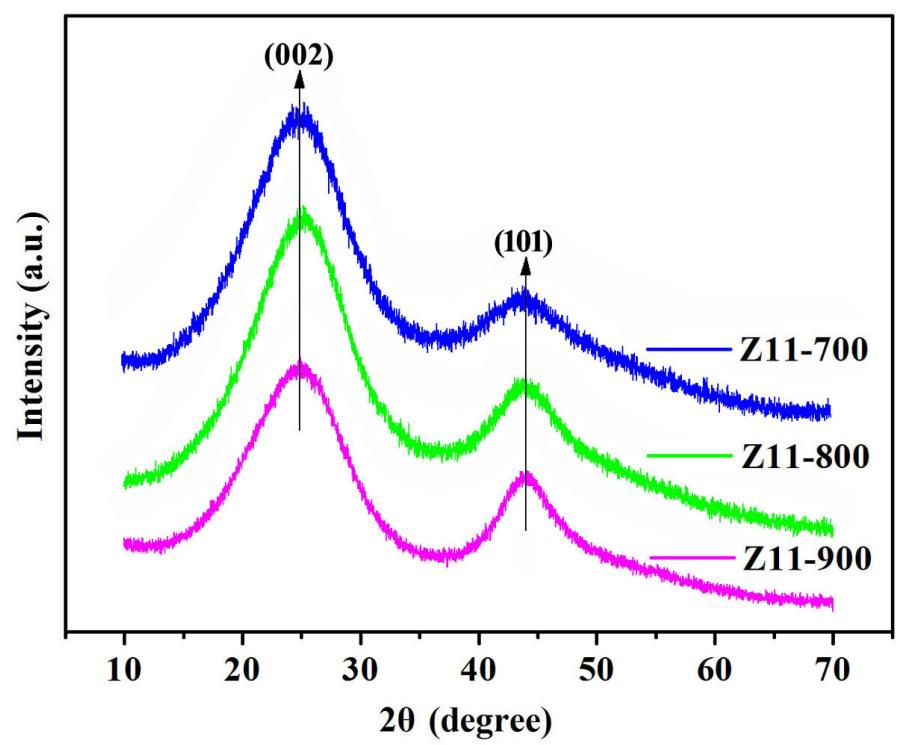

Fig. 2 PXRD patterns of Z11-700, Z11-800 and Z11-900.

FT-IR spectra of Z11-700, Z11-800 and Z11-900 are shown in Fig. 3. The peak at $1620 \mathrm{~cm}^{-1}$ was attributed to the characteristic vibration of $\mathrm{C}=\mathrm{O}$ or $\mathrm{C}=\mathrm{C}$ bonds [44]. The absorption peak which occurred at $1130 \mathrm{~cm}^{-1}$ was related to the $\mathrm{C}-\mathrm{O}$ or $\mathrm{C}-\mathrm{C}$ bonds stretching [45].

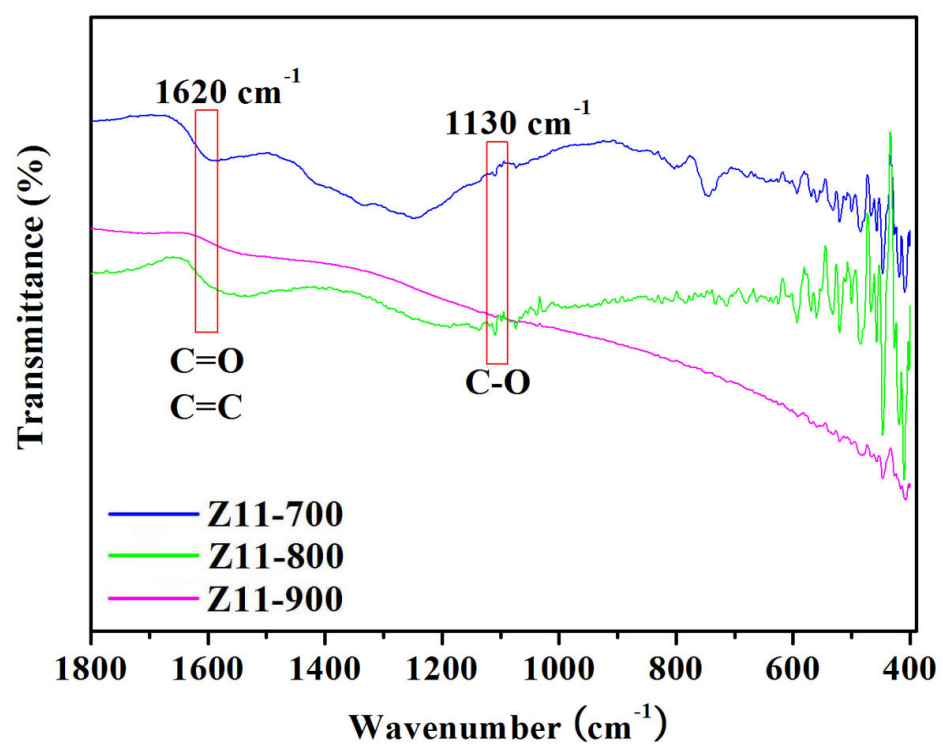

Fig. 3 FT-IR spectra of Z11-700, Z11-800 and Z11-900.

As depicted in Fig. 4, two distinct peaks assigned to D and $G$ bands at about 1350 and $1591 \mathrm{~cm}^{-1}$ were detected in the Raman spectra. The D band represents the 
disordered carbons or defective graphitic structures, and the $\mathrm{G}$ band corresponds to graphitic in-plane vibrations of ideal $\mathrm{sp}^{2}$ carbons [46]. $\mathrm{I}_{\mathrm{D}} / \mathrm{I}_{\mathrm{G}}$, the relative intensity ratio of these two bands, represents the changes of graphitization degree [47]. The value of $\mathrm{I}_{\mathrm{D}} / \mathrm{I}_{\mathrm{G}}$ for the samples Z11-700, Z11-800 and Z11-900 experienced an increase from 0.934, to 0.946 and 0.979 . According to previous reports, this is because of the formation of defective nanocrystalline graphite with the increase of temperature, which manifests in the D band [48].

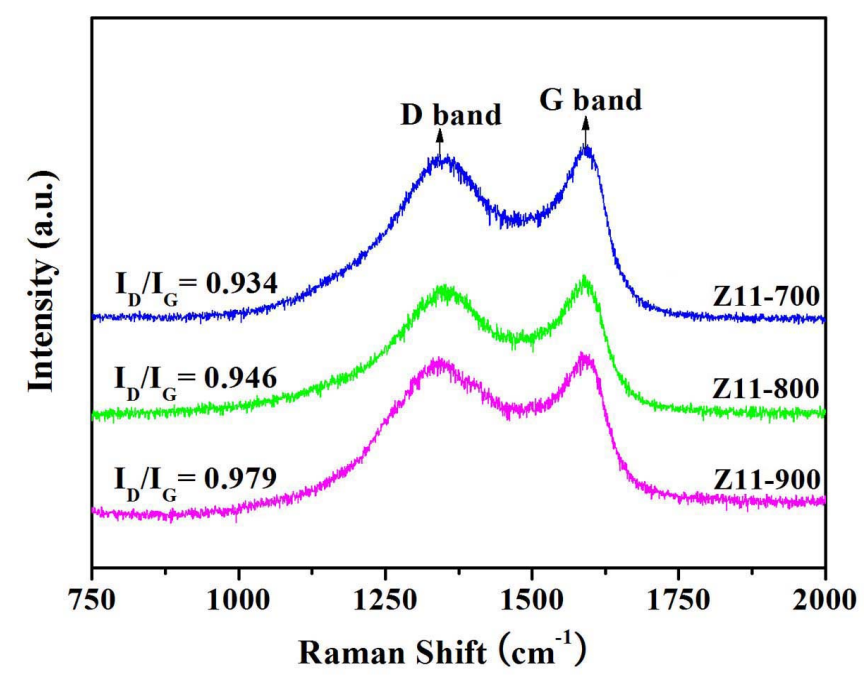

Fig. 4 Raman spectra of Z11-700, Z11-800 and Z11-900.

As shown in Fig. 5a, the nitrogen adsorption-desorption isotherms of Z11-700, Z11-800 and Z11-900 exhibited type I and IV mixed isotherms. The drastic rise and high $\mathrm{N}_{2}$ uptakes at the relative pressure of $\sim 0.5$ illustrated the existence of micropores [49]. The obvious hysteresis loops at the relative pressure of $0.5 \sim 1.0$ can be attributed to the mesoporous porosity [50]. BET surface areas of 377, 260 and 157 $\mathrm{m}^{2} / \mathrm{g}$ for the samples Z11-700, Z11-800 and Z11-900 and their total pore volumes (at $\left.\mathrm{P} / \mathrm{P}_{0}=0.99\right)$ of $0.304,0.299$ and $0.210 \mathrm{~cm}^{3} / \mathrm{g}$ were in the order of $\mathrm{Z} 11-700>\mathrm{Z} 11-800$ $>$ Z11-900, which may lead to a different amount of adsorbate capture [51]. What is more, Z11-700, Z11-800 and Z11-900 displayed a similar micropore size distribution, 
ranging from $0.37 \mathrm{~nm}$ to $0.44 \mathrm{~nm}$, and the total micropore volumes were $0.085,0.092$ and $0.124 \mathrm{~cm}^{3} / \mathrm{g}$, respectively (Fig. 5b). It is noteworthy that Z11-900 with the low surface area and total pore volume had the largest total micropore volume, which maybe lead to a different amount of iodine capture in the sample's micropores [52].
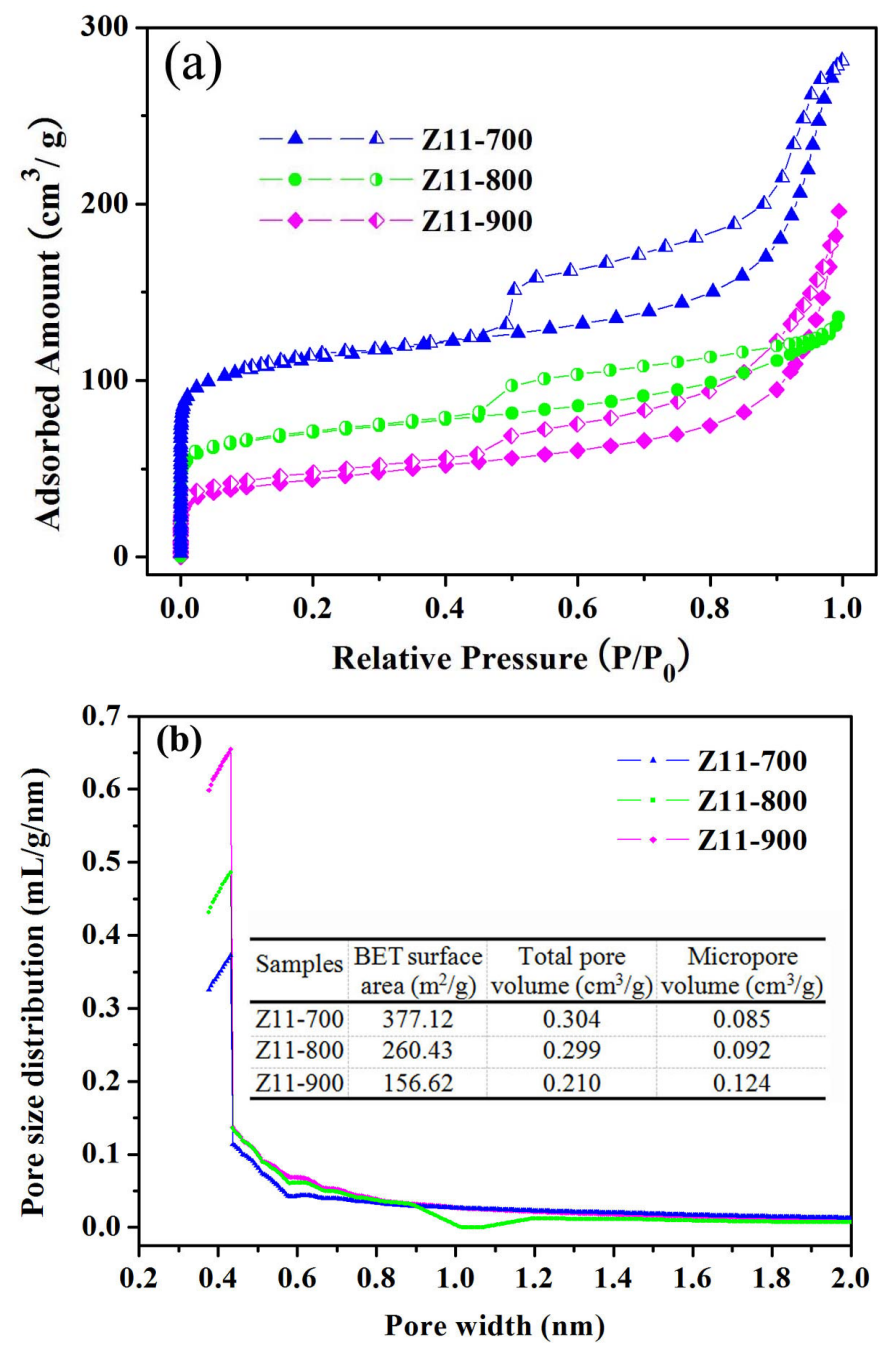

Fig. $5 \mathrm{~N}_{2}$ adsorption-desorption isotherms at $77 \mathrm{~K}$ (a) and micropore size distribution (b) of Z11-700, Z11-800 and Z11-900.

\subsection{Capture of iodine}

The capture of iodine vapour is of great importance and necessity, because any harmful radioisotope release in the environmental can be most detrimental and in particular can directly affect human metabolic processes. In recent years, porous carbon materials are attracting huge interest owing to their environmental friendliness, 
excellent chemical and thermal stabilities, abundant porosity and absorbent capacity, which prompted us to investigate the iodine capture capacity of the synthesized samples. To measure the adsorption properties for iodine, the prepared Z11-700, Z11800 and Z11-900 were exposed to saturated iodine vapour in a sealed container. Gravimetric measurement of the three samples at different time intervals was employed to evaluate the iodine loading. As shown in Fig. 6a and b, the mass of the samples Z11-700, Z11-800 and Z11-900 increased significantly with prolonging the contact time during the initial $10 \mathrm{~h}$. Thereafter, no more changes was observed after $10 \mathrm{~h}$, which indicated that the adsorption for iodine vapour reached equilibrium [53]. The maximum adsorption capacity were 2983, 3255 and $3775 \mathrm{mg} / \mathrm{g}$ for Z11-700, Z11-800 and Z11-900, respectively. Iodine uptake was $2178 \mathrm{mg} / \mathrm{g}$ for ZIF-11. It is obvious that Z11-700, Z11-800 and Z11-900 all experienced higher adsorption capacities than their precursor ZIF-11. Of the three porous carbon samples, Z11-900 exhibited the optimum performance and also exceeded other previously reported porous solid materials.

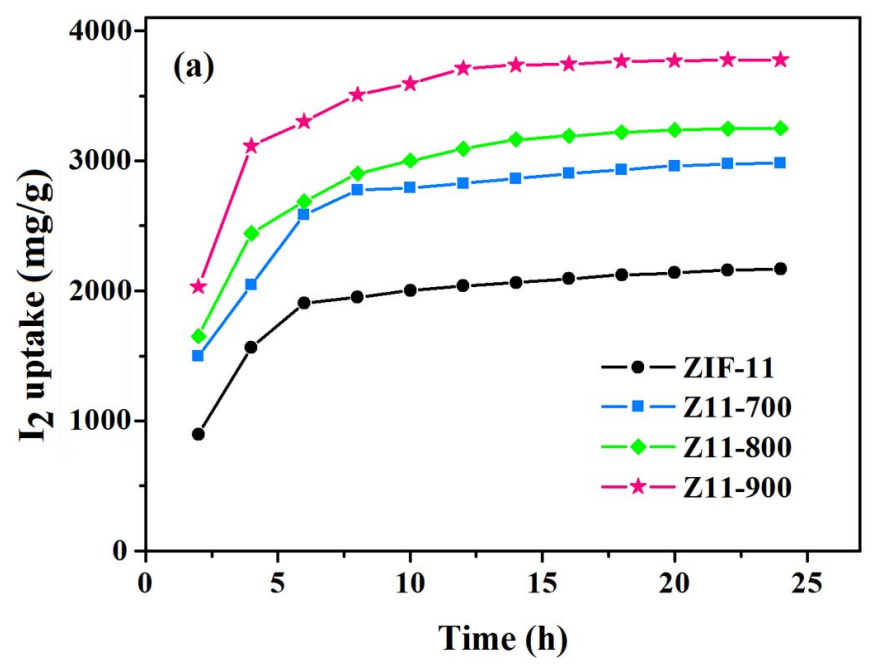




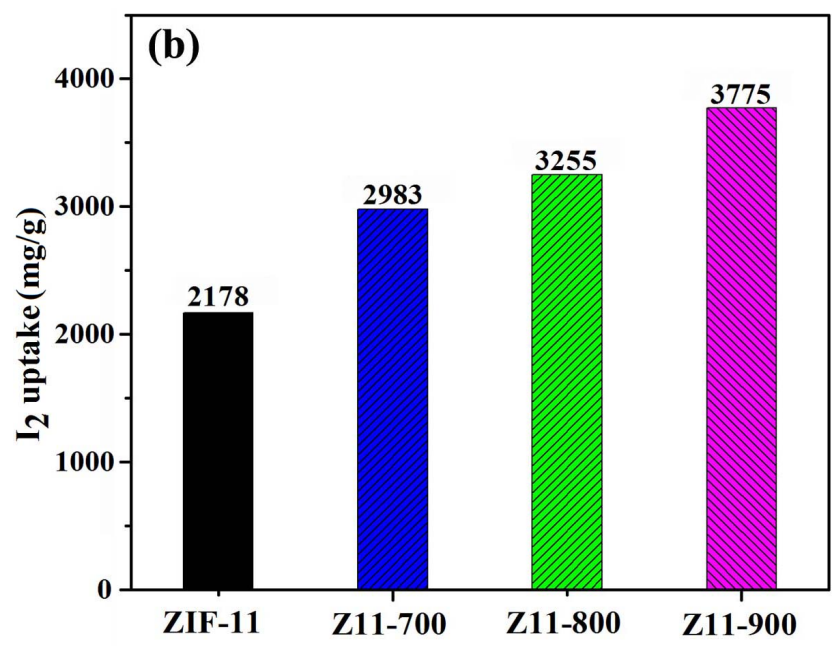

Fig. 6 Iodine vapour uptake $(\mathrm{mg} / \mathrm{g})$ over time at $75^{\circ} \mathrm{C}$ (a) and total uptake comparison (b) for samples ZIF-11, Z11-700, Z11-800 and Z11-900, respectively.

In general, the amount for guest molecules adsorption usually depends on the physical features of the materials such as high specific surface area and porous structure [54]. Among the three samples, although Z11-900 had the lowest surface area and total pore volume, Z11-900 possessed the largest total micropore volume. Abundant micropores together with appropriate micropore size could enhance and stabilize the existance for iodine molecules in Z11-900's pores. It is also accepted that the guest molecules uptake capacity is not only related to the high specific surface area and porous structure, but is associated with the micropore volume [52].

\subsection{Regeneration and reusability of Z11-900}

Regenerability and reusability of exhausted adsorbents are important parameters in practice [55]. In this work, the recovery of Z11-900 saturated with iodine molecules was conducted by immersing the iodine-loaded sample in methanol at room temperature. The desorption was monitored until the amount of the dissolved molecular iodine in the methanol was unchanged. The regenerated Z11-900 was collected by centrifugation, and subsequently washed with fresh methanol four times before being dried overnight for the next evaluation. As shown in Fig. 7, after three 
cycles, the iodine vapour uptake of Z11-900 decreased by $10.3 \%$, but was still maintained at a high level (3672 mg/g). The regeneration efficiency of Z11-700 and Z11-800 were 77.4 and $81.8 \%$ after three cycles, respectively (Figs. S4 and S5). This result illustrated that Z11-900 could be readily and straightforwardly regenerated, and the regenerated sample also exhibited excellent affinity for the iodine vapour. The PXRD patterns of regenerated Z11-900 after three cycles indicated that the structure of the Z11-900 remained unchanged (Fig. S6).

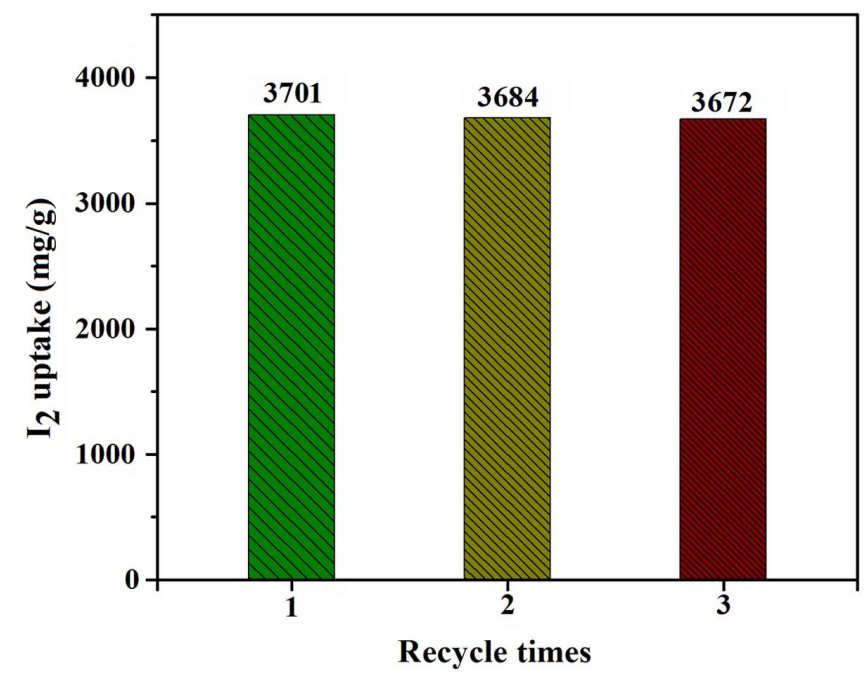

Fig. 7 Recycling of Z11-900 for iodine vapour uptake.

\subsection{Comparison with other porous solid adsorbents}

To evaluate the iodine vapour capture capacity of the synthesized Z11-900, a comparison of the maximum iodine uptake $(\mathrm{mg} / \mathrm{g})$ with other reported adsorbents is shown in Fig 8. The results showed that the adsorption value of Z11-900 was lower than that of TTA-TTB and 2D Meso-COF composites [56, 57]. However, TTA-TTB is a covalent organic framework (COF) which needed $96 \mathrm{~h}$ to achieve adsorption saturation, while Z11-900 only needed 10 h. Z11-900 and KOH-AC had similar adsorption values, but $\mathrm{KOH}-\mathrm{AC}$ was synthesized by using a mixture of activated carbon and $\mathrm{KOH}[18]$. Using alkali was not an optimum option considering the potential environmental impact of the alkali. Z11-900 was far superior compared with 
those reported organic polymers HCMP, CalP4-Li, APOP and carbon materials AK and NT-POP@800 [14,16, 58-60]. At the same time, Z11-900 also had an obvious advantage compared with ZIF-11 and ZIF-8 [34]. This might be mainly attributable to the appropriate micropore size and volume of Z11-900 and the connection between porous carbon materials and target molecules, which facilitated iodine molecular storage.

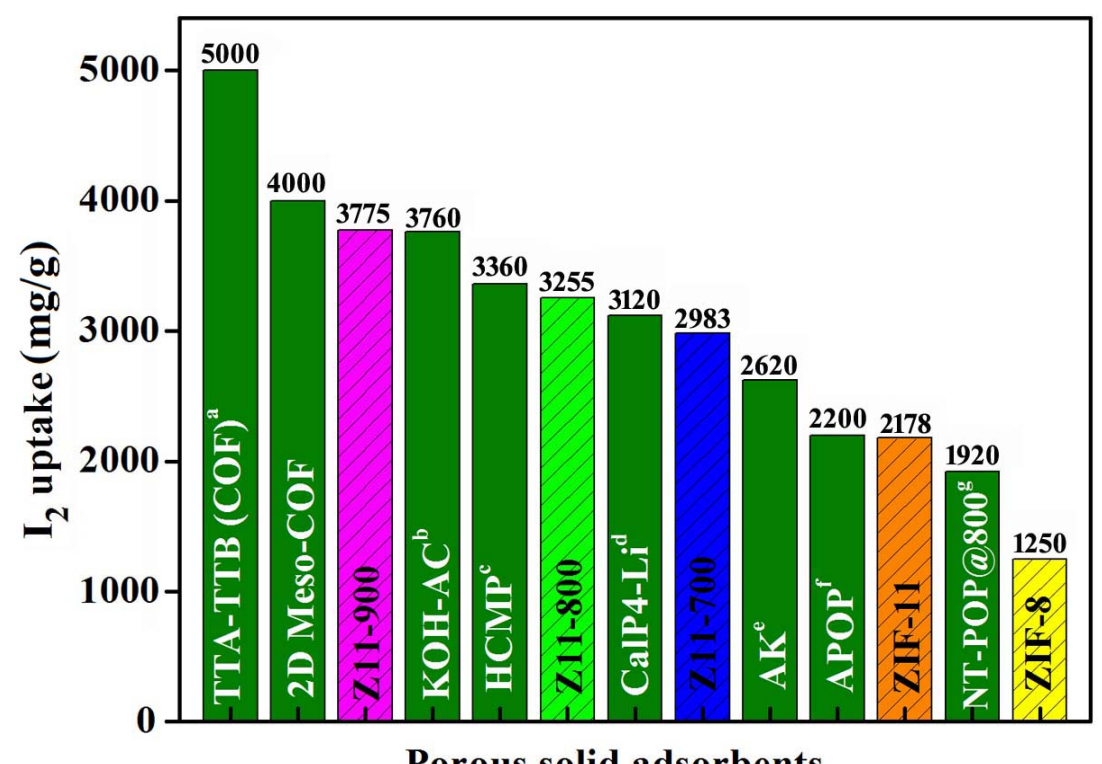

Fig. 8 The comparison of the maximum iodine uptake $(\mathrm{mg} / \mathrm{g})$ with reported adsorbents. a: covalent organic frameworks $(\mathrm{COFs})$; b: carbon materials after carbonizing of activated carbon and a $\mathrm{KOH}$ mixture; c: hexaphenyl benzene-based conjugated microporous polymers (CMPs); d: a calix[4]arene-based porous covalent polymers (POPs); e: carbonized cigarette butts; f: acetal-linked POPs; g: porous carbon materials after carbonization of POPs.

\section{Conclusions}

In this work, three nanoporous carbon materials Z11-700, Z11-800 and Z11-900 were prepared by a one-step calcination of ZIF-11 at 700,800 and $900{ }^{\circ} \mathrm{C}$, respectively. Z11-900 showed an ultrahigh iodine affinity with an adsorption of 3775 $\mathrm{mg} / \mathrm{g}$ at $75{ }^{\circ} \mathrm{C}$, and the uptake value was higher than the template ZIF-11, Z11-700, Z11-800 and some reported solid state porous materials. In addition, Z11-900 could 
be regenerated through simple steps and the regeneration efficiency for iodine vapour is nearly $90 \%$ after three cycles of reuse. Considering the advantages of easy operation, the excellent iodine uptake, especially the recyclable features, ZIF-11 derived nanoporous carbons Z11-900 should be a promising material in environmental applications. Further studies are currently under way in our group to further explore this aspect.

\section{Acknowledgements}

This work was supported by National Natural Science Foundation of China (Grant No. 21471029 and 21601019) and Natural Science Foundation of Changchun Normal University (Grant No. [2017]006).

\section{References}

[1] P. A. Kharecha, J. E. Hansen, Environ. Sci. Technol. 47 (2013) 4889-4895.

[2] X. R. Zhang, I. D. Silva, H. G. W. Godfrey, S. K. Callear, S. A. Sapchenko, Y. Q. Cheng, J. Am. Chem. Soc. 139 (2017) 16289-16296.

[3] X. M. Li, G. Chen, Q. Jia, Micropor. Mesopor. Mat. 279 (2019) 186-192.

[4] S. Liu, N. Wang, Y. Zhang, Y. Li, Z. Han, P. Na, J. Hazard. Mater. 284 (2015) 171181.

[5] K. S. Subrahmanyam, C. D. Malliakas, D. Sarma, G. S. Armatas, J. Wu, M. G. Kanatzidis, J. Am. Chem. Soc. 137 (2015) 13943-13948.

[6] T. C. T. Pham, S. Docao, I. C. Hwang, M. K. Song, D. Y. Choi, D. Moon, P. Oleynikov, K. B. Yoon, Energy Environ. Sci. 9 (2016) 1050-1062.

[7] G. M. Adams, A. S. Weller, Coord. Chem. Rev. 355 (2018) 150-172.

[8] N. Huang, L. Zhai, H. Xu, D. Jiang, J. Am. Chem. Soc. 139 (2017) 2428-2434.

[9] P. A. Kobielska, A. J. Howarth, O. K. Farha, S. Nayak, Coord. Chem. Rev. 358 (2018) 92-107.

[10] K. Shen, L. Zhang, X. Chen, L. Liu, D. Zhang, Y. Han, J. Chen, J. Long, R. Luque, Y. Li, B. Chen, Science, 359 (2018) 206-210.

[11] S. M. Scott, T. Hu, T. K. Yao, G. Q. Xin, J.Lian, Carbon, 90 (2015) 1-8.

[12] B. B. Liu, X. H. Ren, L. Chen, X. X. Ma, Q. Chen, Q. D. Sun, L. Zhang, P.C. 
Si,L. J. Ci, Jour. Haz. Mater. 373 (2019) 705-715.

[13] W. Shuang, A. Li, D. H. Wang, Z. Chang, J. Solid State Chem. 276 (2019) 159163.

[14] H. X. Sun, P. Q. La, R. X. Yang, Z.Q. Zhu, W. D. Liang, B. P. Yang, A. Li, W. Q. Deng, J. Haz. Mater. 321 (2017) 210-217.

[15] M. J. Du, D. He, Y. B. Lou, J. X. Chen, J. Energy Chem. 26 (2017) 673-680.

[16] C. Yao, G. Y. Li, J. K. Wang, Y. H. Xu, L. M. Chang, Sci. Rep. 8 (2018) 18671875.

[17] W. J. Ma, Y. C. Du, N. Wang, P. Miao, Environ. Sci. Pollut. R. 24(2017) 1627616288.

[18] H. X. Sun, P. Q. La, Z. Q. Zhu, W. D. Liang, B. P. Yang, A. Li, J. Mater. Sci. 22 (2015) 7326-7332.

[19] M. Kazemeini, M. Nikkhah, M. Fattahi, L. Vafajoo, Chem. Biochem. Eng. Q. 30 (2016) 9-18.

[20] Y. J. Gong, Y. M. Tang, Z. H. Mao, X. N. Wu, Q. Liu, S. Hu, S. S. Xiong, X. L. Wang, J. Mater. Chem. A 6 (2018) 13696-13704.

[21] R. Shahbazi, A. Payan, M. Fattahi, J. Photoch. Photobio. A 364 (2018) 564-576.

[22] F. Hayati, A. A. Isari, B. Anvaripour, M. Fattahi, B. Kakavandi, Chem. Eng. J. 381 (2020) 122636.

[23] A. Shojaie, M. Fattahi, S. Jorfi, B. Ghasemi, Int. J. Ind. Chem. 9 (2018) 141151.

[24] R. Ashouri, P. Ghasemipoor, B. Rasekh, F. Yazdian, S. R. Mofradnia, M. Fattahi, Int. J. Environ. Sci. Te. 3 (2019) 1729-1740.

[25] M. Fattahi, M. Kazemeini, F. Khorasheh, A. M. Rashidi, Catal. Sci. Technol. 5 (2015) 910-924.

[26] M. Fattahi, M. Kazemeini, F. Khorasheh, A. M. Rashidi, Ind. Eng. Chem. Res. 52 (2013) 16128-16141.

[27] H. Y. Wang and Z. L. Jin, New J.Chem. 42 (2018) 17396-17406.

[28] S. L. Yang, L. Peng, E. Oveisi, S. Bulut, D. T. Sun, M. Asgari, O. Trukhina, W. L. Queen, J. Eur. Chem. 24 (2018) 1-6.

[29] X. W. Zhang, L. Jiang, Z. W. Mo, H. L. Zhou, P. Q. Liao, J. W. Ye, D. D. Zhou and J. P. Zhang, J. Mater. Chem. A 5 (2017) 24263-24268.

[30] A. F. Abdel-Magied, H. N. Abdelhamid, R. M. Ashour, X. D. Zou, K. Forsberg, 
Micropor. Mesopor. Mat. 278 (2019) 175-184.

[31] S. Z. Xu, Y. L. Lv, X. F. Zeng, D. P. Cao, Chem. Eng. J. 323 (2017) 502-511.

[32] C. Yao, D. Cui, Y. Zhu, W. Xie, S. R. Zhang, G. J. Xu, Y. H. Xu, New J. Chem. 43 (2019) 6838-6842.

[33] J. Sánchez-Laínez, B. Zornoza, Á. Mayoral, Ángel. Berenguer-Murcia, D. Cazorla-Amorós, C.Téllez, J. Coronas, J. Mater. Chem. A 3 (2015) 6549-6556.

[34] W. Xie, D. Cui, S. R. Zhang, Y. H. Xu, D. L. Jiang, Mater. Horiz. 6 (2019) 1571-1595.

[35] M. He, J. F. Yao, Q Liu, Z. X. Zhong, H. T. Wang, Dalton Trans. 42 (2013) 16608-16613.

[36] A. Ehsani, M. Pakizeh, J Taiwan Inst. Chem. E 66 (2016) 414-423.

[37] R. Kumar, K. Jayaramulu, T. K. Maji, R. N. C. Rao, Chem. Commun. 49 (2013) 4947-4949.

[38] Z. Li, X. Chen, F. Yu, M. Su, B. Q. Zhang, B. Li, T. L. Zhang, New J. Chem. 40 (2016) 1430-1435.

[39] M. S. Deshmukh, A. Chaudhary, P. N. Zolotarev, R. Boomishankar, Inorg. Chem. 56 (2017) 11762-11767.

[40] B. Reif, C. Paula, F. Fabisch, M. Hartmann, M. Kaspereit, W. Schwieger, Micropor. Mesopor. Mat. 275 (2019) 102-110.

[41] H. Y. Jing, X. D. Song, S. Z. Ren, Y. T. Shi, Y. L. An, Y. Yang, M. Q. Feng, S. B. Ma, C. Hao, Electrochim. Acta 213 (2016) 252-259.

[42] J. L. Huang, F. Hao, X. H. Zhang, J. H. Chen, J. Electroanal. Chem. 810 (2018) 86-94.

[43] F. Hayati, A. A. Isari, M. Fattahi, B. Anvaripour, S. Jorfi, RSC Adv. 8 (2018) 40035-40053.

[44] X. Y. Duan, W. Liu, L. M. Chang, J Taiwan Inst. Chem. E. 62 (2016) 132-139.

[45] X. Y. Cao, J. F. Xia, X. Meng, J. Y. Xu, Q. Y. Liu, Z. H. Wang, Adv. Funct. Mater. 29 (2019) 1902237.

[46] Y. C. Qi, M. L. Yang, W. H. Xu, S. He, Y. Men, J. Colloid Interf. Sci. 486 (2017) 84-96.

[47] B. B. Liu, X. H. Ren, L. Chen, X. X. Ma, Q. Chen, Q. D. Sun, L. Zhang, P. C. Si, L. J. Ci, J. Haz. Mater. 373 (2019) 705-715.

[48] J. C. Zhang, L. H. Liu, G. Qian, Z. J. Zhou, K. S. Xiao, S, Cheng, Y. Wang, Y. F. Liu, Y. Feng, New J. Chem. 43 (2019) 2171-2178. 
[49] F. Hao, L. Li, X. H. Zhang, J. H. Chen, Mater. Res. Bull 66 (2015) 88-95.

[50] M. M. Fan, F. Y. Gai, Y. Cao, Z. B. Zhao, Y. H. Ao, Yu. L. Liu, Q. S. Huo, J. Solid State Chem. 269 (2019) 507-512.

[51] W. Zhou, Y. P. Wu, J. Zhao, W. W. Dong, X. Q. Qiao, D. F. Hou, X. H. Bu, D. S. Li, Inorg. Chem. 56 (2017) 14111-14117.

[52] H. R. Chen, K. Shen, J. Y. Chen, X. D. Chen, Y. W. Li, J. Mater. Chem. A 5 (2017) 9937-9945.

[53] H. X. Sun, B. L. Yang, A. Li, J. Chem. Eng. 372 (2019) 65-73.

[54] X, C, Ma, L. Q. Li, S. B. Wang, M. M. Lu, H. L. Li, W. W. Ma, T. C. Keener, Appl. Surf. Sci. 369 (2016) 390-397.

[55] G. B. Hong, Y. K. Wang, Appl. Surf. Sci. 423 (2017) 800-809.

[56] P. Wang, Q. Xu, Z. Li, W. Jiang, Q. Jiang, D. Jiang, Adv. Mater. 30 (2018) 1801991-1801997.

[57] S. H. An, X. Zhu, Y. Y. He, L. Yang, H. Wang, S. B. Jin, J. Hu, H. L. Liu, Ind. Eng. Chem. Res. 24 (2019) 10495-10502.

[58] Y. Z. Liao, J. Weber, B. M. Mills, Z. H. Ren, C. F. J. Faul, Macromolecules 49 (2016) 6322-6333.

[59] B. X. Guo, S. J. Wu, Q. Su, W. T. Liu, P. Y. Ju, G. H. Li, Q. L. Wu, Mater. Lett. 229 (2018) 240-243.

[60] D. F. Sava, M. A. Rodriguez, K. W. Chapman, P. J. Chupas, J. A. Greathouse, P. S. Crozier, T. M. Nenoff, J. Am. Chem. Soc. 133 (2011) 12398-12401. 